\title{
Caracterización de las potenciales interacciones farmacológicas en pacientes de una unidad de cuidados intensivos en un hospital de tercer nivel de Bogotá
}

\author{
Marcela Hernández, Gabriel Tribiño, Carlos Bustamante \\ Departamento de Farmacología Clínica y Terapéutica, Universidad de La Sabana, Chía, Colombia
}

\begin{abstract}
Introducción. Las interacciones farmacológicas ocurren con mayor frecuencia en las unidades de cuidados intensivos que en otros servicios. Sin embargo, en Colombia son pocos los estudios en que se han evaluado en pacientes críticamente enfermos.

Objetivos. Caracterizar las potenciales interacciones farmacológicas en las prescripciones de pacientes hospitalizados en una unidad de cuidados intensivos y determinar los factores asociados con su aparición.

Materiales y métodos. Se analizó una cohorte retrospectiva de pacientes hospitalizados en una unidad de cuidados intensivos, durante un periodo de siete meses. Las prescripciones diarias se evaluaron en busca de potenciales interacciones farmacológicas mediante el programa Lexicomp ${ }^{\mathrm{TM}}$. Se calculó la incidencia de interacciones, se clasificaron según su tipo, gravedad y grado de documentación, y se evaluaron los factores asociados con su aparición mediante regresión logística.

Resultados. La proporción de pacientes con por lo menos una interacción fue de $84 \%$, en tanto que el $87 \%$ presentó más de una interacción; la mediana fue de seis interacciones por paciente. La más frecuente fue entre el fentanilo y el midazolam (23\%). Las interacciones de gravedad y grado de documentación moderados fueron las más frecuentes ( 77,6 y $52,6 \%$, respectivamente). El fármaco índice más frecuente fue el midazolam (12\%) y el precipitante más frecuente, el fentanilo (10,6\%). La edad (odds ratio, $\mathrm{OR}=3,1)$ y el número de medicamentos $(\mathrm{OR}=11,8)$, se asociaron con la aparición de interacciones.

Conclusiones. Dada su elevada frecuencia y potencial impacto negativo es importante vigilar sistemáticamente las prescripciones en cuidados intensivos para detectar las interacciones, con el fin de contribuir al uso racional de los medicamentos y a mejorar la calidad de la atención.
\end{abstract}

Palabras clave: interacciones medicamentosas; cuidados críticos; prescripciones de medicamentos; incidencia; factores de riesgo; estudios de cohortes.

doi: https://doi.org/10.7705/biomedica.v38i4.3884

Characterization of potential drug-drug interactions in patients hospitalized in the intensive care unit of a tertiary hospital in Bogotá

Introduction: Drug-drug interactions occur more frequently in intensive care units than in other services. However, in Colombia, there are few studies on this problem in critically ill patients.

Objectives: To characterize potential drug-drug interactions generated from prescriptions during hospitalization in an intensive care unit and to determine factors associated with their onset.

Materials and methods: A retrospective cohort was assembled with patients hospitalized in an intensive care unit for a seven-month period. The daily prescription was assessed for potential drugdrug interactions using the Lexicomp ${ }^{\circledR}$ program. We calculated the incidence of interactions, classified them by type, severity, and level of documentation, and evaluated the factors associated with their onset using logistic regression.

Results: The proportion of patients with at least one interaction was $84 \%$ while $87 \%$ had more than one interaction; the median was six interactions per patient. The most frequent was fentanyl and midazolam (23\%). Moderate interactions were the most frequent by severity $(77.6 \%)$ and by documentation (52.6\%). The most common index and precipitating drugs were midazolam (12\%) and fentanyl $(10.6 \%)$, respectively. Age $(\mathrm{OR}=3.1)$ and the number of drugs $(\mathrm{OR}=11.8)$ were associated with the occurrence of interactions.

\section{Contribución de los autores:}

Marcela Hernández: elaboración del protocolo y recolección de la información

Gabriel Tribiño: asesoría para la metodología en la elaboración del protocolo y del análisis estadístico (concepción y presentación)

Carlos Bustamante: asesoría en la elaboración del protocolo desde el punto de vista temático

Todos los autores participaron en la escritura del manuscrito. 
Conclusions: Given their high frequency and potential negative impact, the systematic monitoring of prescriptions in intensive care units to detect interactions is important. Such monitoring contributes to the rational use of medicines and to improve the quality of care.

Key words: Drug interactions; critical care; drug prescriptions; incidence; risk factors; cohort studies. doi: https://doi.org/10.7705/biomedica.v38i4.3884

El tema de las interacciones farmacológicas recibe cada vez mayor atención como consecuencia del creciente desarrollo de nuevos medicamentos durante los últimos 20 años (1). Estas interacciones son frecuentes, pueden impactar de forma negativa los resultados en salud y son parte del $70,4 \%$ de los problemas prevenibles relacionados con medicamentos. Además, la proporción de reacciones adversas prevenibles causadas por interacciones oscila entre 35 y $81 \%(2,3)$.

En una revisión de la literatura, Becker, et al. (4), encontraron que $0,57 \%$ de los ingresos hospitalarios y $0,12 \%$ de los reingresos se debían a interacciones farmacológicas y, aunque el número puede parecer modesto, resulta significativo si se tiene en cuenta el promedio general de hospitalizaciones y reingresos (4). En pacientes hospitalizados, se ha reportado que entre el 3 y el $5 \%$ de las reacciones adversas prevenibles pueden deberse a interacciones farmacológicas $(3,4)$. En diversos estudios, la prevalencia de tales interacciones en las unidades de cuidados intensivos ha oscilado entre el 11 y el $85 \%(1,5,6)$, dependiendo del número de pacientes incluidos y los sistemas de verificación que se empleen para detectarlas y clasificarlas según su gravedad.

Son pocos los estudios que se han llevado a cabo sobre la relevancia clínica de tales interacciones. En 2011, Reis, et al., encontraron que estas se habían presentado en el $70 \%$ de las prescripciones evaluadas, $23 \%$ de las cuales fueron clínicamente relevantes (7). Sin embargo, según el método de clasificación que se utilice, esta última proporción puede disminuir a $12 \%(8,9)$. Entre las consecuencias derivadas de las interacciones farmacológicas, se encuentran las complicaciones de las condiciones de base, el incremento de la estancia hospitalaria y el aumento en la mortalidad $(10,11)$. Según los National Institutes of Health

\section{Correspondencia:}

Marcela Hernández, Departamento de Farmacología Clínica y Terapéutica, Universidad de La Sabana, Chía, Colombia Teléfono: (310) 8006559

marcela.herlon@outlook.com

Recibido: 26/04/17; aceptado: 21/03/18 de los Estados Unidos, ocurren entre 44.000 y 98.000 muertes al año a causa de errores médicos (12), de las cuales 7.000 son el resultado de reacciones adversas a medicamentos, y de estas, cerca de 6,9 \% corresponden a interacciones farmacológicas (13).

Por otra parte, los pacientes en mayor riesgo de presentar interacciones medicamentosas son: los que reciben varios fármacos a la vez, los infectados con HIV, los que han recibido trasplantes y los críticamente enfermos (14). Estos últimos pueden llegar a recibir hasta 15 medicamentos simultáneamente $y$, además, presentan cambios en la farmacocinética que contribuyen a aumentar el riesgo (1).

En Colombia, la investigación de las interacciones farmacológicas sigue siendo limitada. Hay estudios sobre grupos farmacológicos particulares, pero, en un contexto más amplio y en unidades de cuidados intensivos, son escasas. La falta de información en este campo específico impide detectar oportunamente un problema que aumenta la morbimortalidad, el cual podría prevenirse mediante herramientas validadas que están disponibles para el personal de salud y permiten la prescripción más racional y segura de medicamentos.

El objetivo de este estudio fue detectar las interacciones entre los medicamentos prescritos durante la estancia en una unidad de cuidados intensivos y, además, caracterizar su frecuencia, tipo, gravedad, grado de documentación y fármacos más frecuentemente implicados; asimismo, evaluar la asociación entre dichas interacciones y las variables de edad, sexo, número de medicamentos prescritos, número de prescriptores y días de estancia.

\section{Materiales y métodos \\ Tipo de estudio y población}

Se hizo un estudio retrospectivo de cohorte. La cohorte se seleccionó de los pacientes hospitalizados en la unidad de cuidados intensivos de una clínica de tercer nivel de un municipio del departamento de Cundinamarca (Colombia), entre el $1^{\circ}$ de junio y el 31 de diciembre de 2013; esta unidad cuenta con 22 camas. 
Se incluyeron los pacientes mayores de 18 años de cualquier sexo, hospitalizados por cualquier causa, con una estancia mayor o igual a 24 horas, a quienes se les habían prescrito, por lo menos, dos medicamentos durante el periodo de estudio. Se excluyeron los pacientes que recibían únicamente formulaciones de nutrición parenteral o entérica.

\section{Detección y caracterización de las interacciones farmacológicas}

Para la detección, se revisó la prescripción diaria de cada paciente durante su estancia en la unidad según el registro en la base de datos de la institución, a cargo exclusivamente del personal médico de la unidad.

Se aplicó el identificador de interacciones del programa Lexicomp ${ }^{\mathrm{TM}}$, versión 2014, desarrollado por el Grupo Wolters Kluwer. La identificación y la clasificación de las interacciones generadas por esta herramienta, no difieren significativamente de las obtenidas mediante Micromedex ${ }^{\mathrm{TM}}$ (15). Con Lexicomp ${ }^{\mathrm{TM}}$, las interacciones farmacológicas se clasifican según el grado de documentación, como excelentes, buenas, deficientes o desconocidas; según la gravedad, como menores, moderadas, mayores o contraindicadas (cuadro 1); según el tiempo de aparición, como tempranas ( $\leq 24$ horas) o tardías (>24 horas), y según su tipo, como farmacocinéticas, farmacodinámicas o mixtas (16).

También, se registraron el tiempo de aparición de la primera interacción y el tiempo de exposición en días. Los datos sobre edad, sexo, diagnóstico de ingreso, estancia hospitalaria, número de prescriptores y total de medicamentos prescritos también se extrajeron de la base de datos de la clínica, suministrada en formato de libro de Excel ${ }^{\mathrm{TM}}$ para su análisis. La base de datos contenía la información de la historia clínica electrónica, en la cual las formulaciones diarias registradas tienen un enlace con el departamento de farmacia hospitalaria.

\section{Análisis de datos}

Las medidas de frecuencia calculadas fueron las siguientes.

1. Tasa de incidencia (número de pacientes con una o más interacciones, sobre la suma de personadía de estancia en la unidad de cuidados intensivos). Para los pacientes que presentaron varias interacciones, el denominador se calculó teniendo en cuenta el tiempo desde el ingreso a la unidad de cuidados intensivos hasta el momento en el que se presentó la primera interacción.

2. Proporción de pacientes con una o más interacciones (pacientes con interacciones, sobre número total de pacientes).

3. Proporción de pacientes con dos o más interacciones (número de pacientes con dos o más, sobre número de pacientes con una o más.

4. Tasa absoluta (número total de interacciones/ duración global en días del periodo de observación).

5. Proporción de prescripciones con una o más interacciones sobre el número total de prescripciones).

Los pacientes con interacciones y sin ellas se compararon en cuanto a edad, número de medicamentos recibidos y tiempo de estancia mediante la prueba $t$ de Student, y en cuanto a sexo y morbilidad, mediante la prueba de ji al cuadrado. También, se compararon los pacientes con por lo menos una interacción mayor y aquellos con interacciones moderadas o leves, empleando las mismas variables y pruebas estadísticas.

Los factores asociados con las interacciones se evaluaron mediante un análisis de regresión logística en el que la variable dependiente fue la presencia de por lo menos una interacción y las variables independientes, la edad, el sexo, el número de prescriptores, la duración de la estancia

Cuadro 1. Clasificación de interacciones según el programa Lexicomp ${ }^{\mathrm{TM}}$

\begin{tabular}{|c|c|c|}
\hline $\begin{array}{l}\text { Categoría de } \\
\text { gravedad }\end{array}$ & Definición de gravedad & Nivel de documentación \\
\hline $\mathrm{X}$ & Contraindicada & Excelente: comprobada con buenos estudios clínicos \\
\hline $\mathrm{D}$ & Mayor: puede causar daño o requerir manejo. & $\begin{array}{l}\text { Buena: bien documentada, y es conocida. } \\
\text { No validada por estudios clínicos }\end{array}$ \\
\hline $\mathrm{C}$ & $\begin{array}{l}\text { Moderada: puede exacerbar la condición clínica o } \\
\text { requerir modificaciones en el tratamiento. }\end{array}$ & $\begin{array}{l}\text { Moderada: hay datos sugestivos. Buena } \\
\text { documentación para un fármaco similar }\end{array}$ \\
\hline $\mathrm{B}$ & $\begin{array}{l}\text { Menor: puede tener mínimos efectos clínicos y no } \\
\text { requerir modificación. }\end{array}$ & $\begin{array}{l}\text { Deficiente: datos muy limitados, pero teóricamente } \\
\text { posible }\end{array}$ \\
\hline A & No se conoce. & No se conoce. \\
\hline
\end{tabular}


y el número de medicamentos. Inicialmente, se hizo un análisis bivariado y, luego, las variables que presentaron un valor de $p$ menor de 0,05 se incluyeron en un análisis de regresión logística múltiple. En este análisis no se incluyeron las interacciones clasificadas como deficientes. Dado que los pacientes con interacciones intencionadas pero con potencial de riesgo, pueden representar un grupo etiológicamente diferente, se hizo un análisis de sensibilidad repitiendo los análisis de regresión sin tener en cuenta a dichos sujetos.

El tamaño de la muestra se calculó para establecer la asociación mediante el enfoque de casos por variable independiente. Tomando 12 casos por variable independiente, se requerían 84 casos de interacción farmacológica. Se asumió una frecuencia de pacientes con por lo menos una interacción del $50 \%$, una frecuencia de pacientes con interacciones deficientes del $10 \%$ y una frecuencia de pacientes con interacciones benéficas pero con riesgo potencial del $10 \%$ (los cuales no se tuvieron en cuenta en el análisis de sensibilidad).

Así, con un $30 \%$ de pacientes con interacciones farmacológicas elegibles para el análisis de sensibilidad, se requería un total de 280 pacientes, a lo cual se sumó un $10 \%$ de no elegibles, para un tamaño de muestra de 308. Dado que el periodo durante el cual se atendió un número de sujetos igual o superior al tamaño de muestra calculado fue entre el $1^{\circ}$ de junio y el 31 de diciembre del 2013, se decidió evaluar a todos los pacientes hospitalizados en la unidad de cuidados intensivos durante ese periodo $(\mathrm{N}=356)$. Con el número de pacientes que resultaron elegibles $(\mathrm{N}=312)$, se obtenía un poder mayor de $80 \%$ para encontrar asociaciones estadísticamente significativas con una razón de momios (OR, Odds Ratio) mayor de 2. La base de datos se elaboró en Excel ${ }^{\mathrm{TM}}$, versión 2013, y el análisis estadístico se hizo con el programa Stata $12^{\mathrm{TM}}$.

El estudio se sometió a consideración del comité de investigación y ética de la institución y se obtuvo la aprobación para el uso de su base de datos, la cual fue entregada a los investigadores por un funcionario del departamento de informática designado por el comité. Los resultados se presentaron a la institución, la cual estableció planes de farmacovigilancia activa y brindó educación a la comunidad médica, así como un mejor acceso a las herramientas disponibles para evaluar las interacciones con la intervención activa del departamento de farmacología clínica.

\section{Resultados}

En el estudio se incluyeron 312 pacientes. Las causas de exclusión se presentan en la figura 1 y, las características de los pacientes, en el cuadro 2. En 263 pacientes $(84 \%)$ se presentó, por lo menos, una interacción. La tasa de incidencia fue de 5,8 por 10 persona-día de estancia en la unidad de cuidados intensivos. La proporción de pacientes con más de una interacción fue de $87 \%$ (229/263) y, en estos, la mediana del número de interacciones fue de seis (rango intercuartílico: 6-9). La tasa absoluta fue de siete interacciones por día, la mediana de tiempo para la primera interacción fue un día (rango intercuartílico: 1-2) y la mediana de tiempo de exposición a la interacción fue de cuatro días (rango intercuartílico: 3-9).

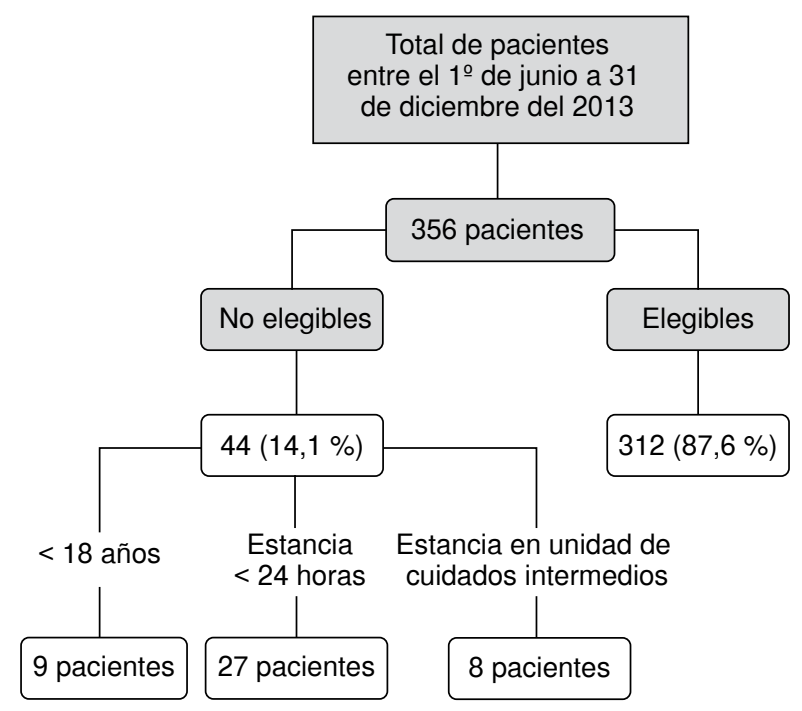

Figura 1. Flujograma de pacientes

Cuadro 2. Caracterización de la población estudiada $(n=312)$

\begin{tabular}{|c|c|c|}
\hline Variable & \multicolumn{2}{|c|}{ Medida de resumen } \\
\hline Sexo masculino, n (\%) & \multicolumn{2}{|c|}{$169(54,1)$} \\
\hline Edad (años) (media $\left.\pm \mathrm{DE}^{*}\right)$ & \multicolumn{2}{|c|}{$55,09 \pm 21,6$} \\
\hline $\begin{array}{l}\text { Tiempo de estancia (días) } \\
\text { (media } \pm D E)\end{array}$ & \multicolumn{2}{|c|}{$5,5 \pm 3,98$} \\
\hline $\begin{array}{l}\text { Medicamentos prescritos por } \\
\text { paciente (mediana, RI) }\end{array}$ & \multicolumn{2}{|c|}{$17(11-22)$} \\
\hline $\begin{array}{l}\text { Número de prescriptores por } \\
\text { paciente (mediana, RI) }\end{array}$ & \multicolumn{2}{|c|}{$4(3-6)$} \\
\hline Grupos de diagnósticos de & \multicolumn{2}{|c|}{ Cardiovascular $96(30,8)$} \\
\hline \multirow[t]{5}{*}{ ingreso más frecuentes, $\mathrm{n}(\%)$} & Neurológico & $78(25)$ \\
\hline & Infeccioso & $57(18,3)$ \\
\hline & Metabólico & $24(7,7)$ \\
\hline & Renal & $16(5,1)$ \\
\hline & Ortopedia & $6(1,9)$ \\
\hline
\end{tabular}

DE: desviación estándar; RI: rango intercuartílico 
Se evaluaron 5.315 prescripciones, de las cuales $1.514(28,5 \%)$ presentaron por lo menos una interacción, con una mediana de interacciones por prescripción de cinco (rango intercuartílico: 2-8). Las características de las interacciones detectadas se presentan en el cuadro 3. La interacción más frecuente fue la de fentanilo con midazolam, seguida por la de enoxaparina con potasio, y omeprazol con losartán. Las diez interacciones más frecuentes se resumen en la figura 2. Los fármacos precipitantes más frecuentes fueron el fentanilo y la dexametasona, y los fármacos índice más frecuentes, el midazolam y el fentanilo (figuras 3 y 4 ).

Según la gravedad, las interacciones moderadas fueron las más frecuentes (con la combinación de fentanilo y midazolam, en primer lugar), seguidas

Cuadro 3. Caracterización de las interacciones detectadas

\begin{tabular}{|c|c|c|}
\hline Característica & $\mathbf{n}$ & $\%$ \\
\hline \multicolumn{3}{|l|}{ Presentación } \\
\hline Temprana ( $\leq 24$ horas) & 903 & 59,7 \\
\hline Tardía (>24 horas) & 611 & 40,3 \\
\hline \multicolumn{3}{|l|}{ Tipo de interacción } \\
\hline Farmacodinámica & 893 & 55,5 \\
\hline Farmacocinética & 560 & 37,0 \\
\hline Mixta & 25 & 1,7 \\
\hline Desconocida & 35 & 2,3 \\
\hline \multicolumn{3}{|l|}{ Gravedad } \\
\hline Mayor & 337 & 22,2 \\
\hline Moderada & 1.175 & 77,6 \\
\hline \multicolumn{3}{|l|}{ Documentación } \\
\hline Excelente & 82 & 5,4 \\
\hline Buena & 611 & 40,3 \\
\hline Moderada & 797 & 52,6 \\
\hline Deficiente & 24 & 1,6 \\
\hline \multicolumn{3}{|c|}{ Tiempo de exposición (días) } \\
\hline$\leq 2$ & 305 & 20,1 \\
\hline 3 a 5 & 929 & 61,4 \\
\hline$>5$ & 280 & 18,5 \\
\hline Interacciones benéficas & 176 & 11,6 \\
\hline
\end{tabular}

por las mayores (con la combinación de fenitoína y midazolam, en primer lugar). Las diez interacciones moderadas o mayores más frecuentes se presentan en el cuadro 4 . No se detectaron interacciones menores ni contraindicadas.

En cuanto al grado de documentación, la mayor proporción de interacciones se clasificaron como moderadas $(n=797)$, las tres más frecuentes fueron las de enoxaparina y cloruro de potasio $(n=35$; $4,4 \%)$, omeprazol y losartán $(n=33 ; 4,1 \%)$ y bromuro de ipratropio y fenitoína ( $n=23 ; 2,9 \%)$. Las tres interacciones con buena documentación más frecuentes fueron: fentanilo y midazolam ( $n=71 ; 11,6 \%)$, fentanilo y propofol ( $n=18 ; 2,9 \%)$, y fentanilo y morfina $(n=16 ; 2,6 \%)$. Las interacciones con documentación excelente fueron las menos frecuentes $(n=82)$; entre estas, la de mayor frecuencia fue la de gluconato de calcio y amlodipino ( $n=12 ; 14,6 \%)$, seguida por la de claritromicina y midazolam $(n=6 ; 7,3 \%)$ y la de nimodipino y fenitoína $(n=6 ; 7,3 \%)$.

Al comparar a los pacientes que presentaron interacciones con quienes no, se encontró que los primeros eran de mayor edad (media de 57,1 Vs. 43,8 años; $p=0,0001$ ), recibieron un mayor número de medicamentos (media 18,5 Vs. 9,3; $p=0,000$ ), en la formulación intervino un mayor número de prescriptores (media 4,9 Vs. 3,1; $p=0,000$ ) y su estancia fue más prolongada (media 6 Vs. 2,9 días; $p=0,000$ ). Las diferencias en cuanto al sexo y los grupos de diagnóstico de ingreso no fueron estadísticamente significativas.

Al comparar los pacientes que presentaron interacciones mayores con quienes presentaron interacciones moderadas, se encontró una diferencia estadísticamente significativa para las variables

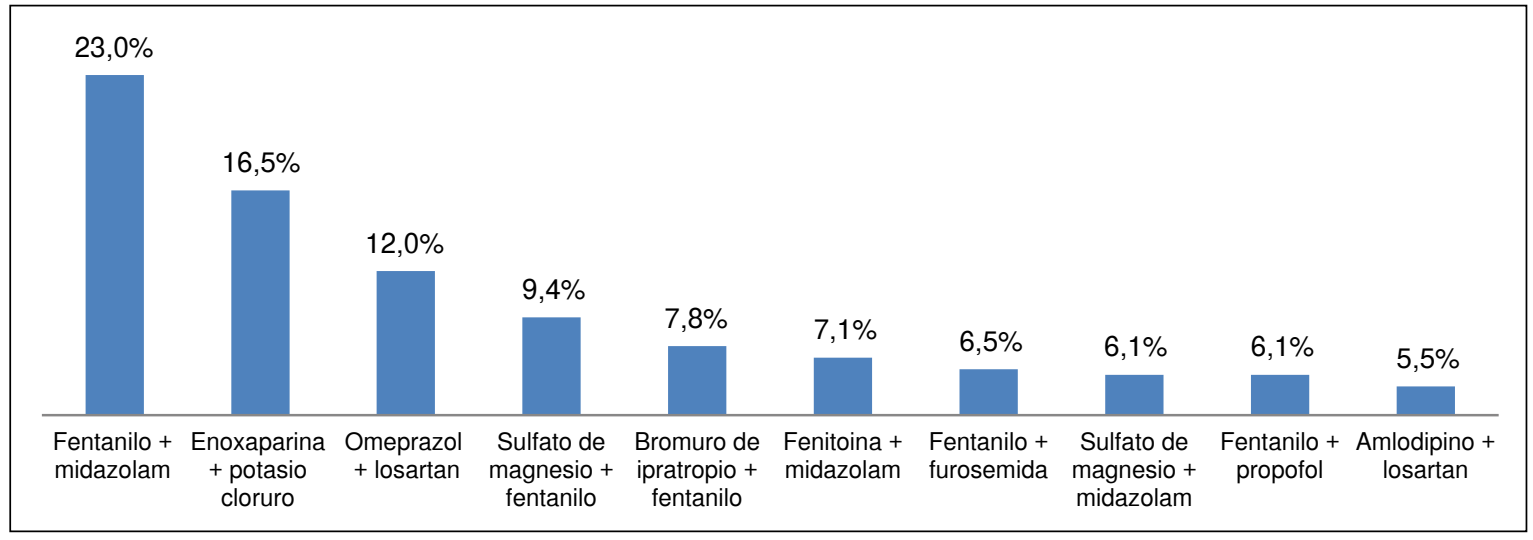

Figura 2. Interacciones más frecuentes 


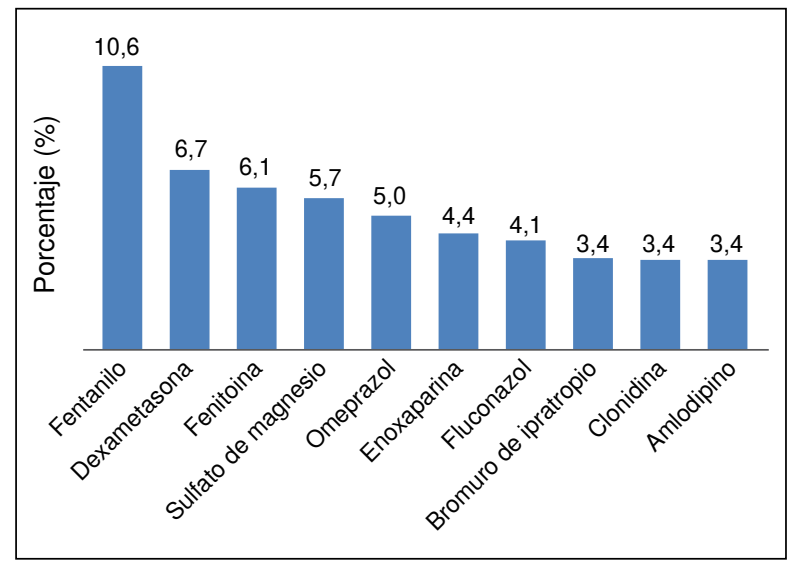

Figura 3. Fármacos precipitantes más frecuentes en las interacciones detectadas $(n=1.514)$

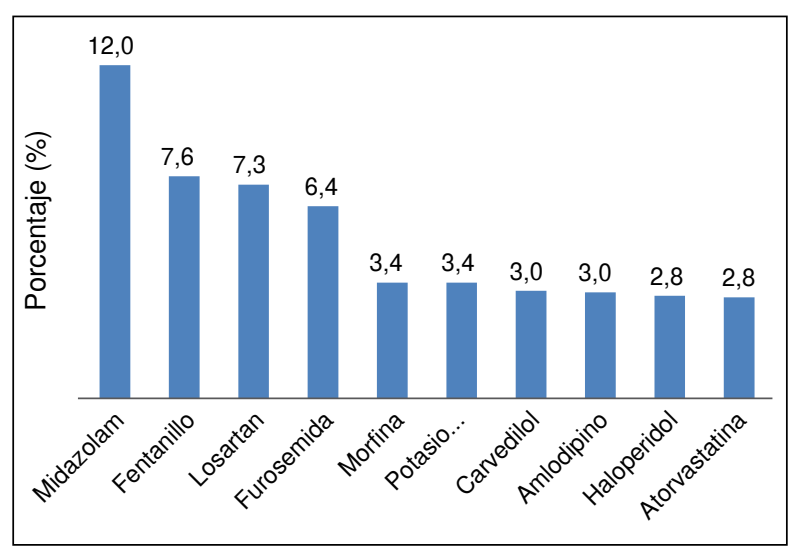

Figura 4. Fármacos índice más frecuentes en las interacciones detectadas $(n=1.514)$

de número de medicamentos recibidos $(20,5 \mathrm{Vs}$. $16,4$, respectivamente; $p=0,000)$, de número de prescriptores (media 5,5 Vs. 4,4; $p=0,00$ ) y de duración de la estancia en días (media 7,6 Vs. $4,5 ; p=0,00)$. No se encontraron diferencias estadísticamente significativas en cuanto a la edad y el sexo.

En el análisis bivariado se encontró que una edad mayor de 60 años, un número de prescriptores mayor de cuatro, una estancia mayor de tres días, y recibir más de diez medicamentos, aumentaban en forma significativa la posibilidad de que se presentara, por lo menos, una interacción (cuadro 5). Sin embargo, al introducir estas variables en el análisis de regresión múltiple, solamente la edad y el número de medicamentos prescritos retuvieron la significación. El ser mayor de 60 años aumentó la posibilidad de que se presentara, por lo menos, una interacción en 3,1 veces, en tanto que recibir
Cuadro 4. Interacciones mayores y moderadas más frecuentes

\begin{tabular}{lrr}
\hline & $\mathbf{n}$ & $\%$ \\
\hline Gravedad mayor (n=337) & & \\
Fenitoína + midazolam & 21 & 6,2 \\
Dexametasona + midazolam & 16 & 4,7 \\
Omeprazol + atorvastatina & 15 & 4,5 \\
Fluconazol + fentanilo & 12 & 3,6 \\
Metoclopramida + haloperidol & 12 & 3,6 \\
Dexametasona + haloperidol & 9 & 2,7 \\
Fenitoina + dexametasona & 9 & 2,7 \\
Dexametasona + losartan & 8 & 2,4 \\
Haloperidol + fentanilo & 8 & 2,4 \\
Claritromicina + fentanilo & 6 & 1,8 \\
Diez más frecuentes & 116 & 34,4 \\
Gravedad moderada (n=1.175) & & \\
Fentanilo + midazolam & 69 & 5,9 \\
Enoxaparina + potasiocloruro & 37 & 3,1 \\
Omeprazol + losartan & 37 & 3,1 \\
Sulfato de magnesio + fentanilo & 29 & 2,5 \\
Bromuro de ipratropio + fentanilo & 24 & 2,0 \\
Fentanilo + furosemida & 20 & 1,7 \\
Sulfato de magnesio + midazolam & 19 & 1,6 \\
Fentanilo + propofol & 18 & 1,5 \\
Amlodipino + losartan & 16 & 1,4 \\
Fentanilo + morfina & 16 & 1,4 \\
Diez más frecuentes & 285 & 24,3 \\
\hline
\end{tabular}

entre 11 y 20 medicamentos la aumentó en 11,8 veces (cuadro 6). En el análisis de sensibilidad, el cual no incluyó las interacciones favorables, se encontraron asociaciones estadísticamente significativas con las mismas variables, aunque con efectos de menor magnitud.

\section{Discusión}

En este estudio la proporción de pacientes con interacciones farmacológicas se ubicó en el rango reportado en otros $(53-87,9 \%)(1,6,7)$. Sin embargo, debe tenerse en cuenta que las metodologías de dichos estudios difieren: en algunos, como el de Caribé, et al. (5), se hizo una evaluación prospectiva y observacional en cohortes pequeñas (86 pacientes), en tanto que, en otros, como el de Carvalho (17), se estimó la prevalencia en momentos específicos de la hospitalización y en grupos grandes (1.124 pacientes); además, la sensibilidad y la especificidad de los programas utilizados para detectar las interacciones también variaba, todo lo cual dificulta la comparación de sus resultados. Un factor que pudo contribuir a la gran frecuencia de interacciones hallada en el presente estudio, es que Lexicomp ${ }^{\mathrm{TM}}$ es de los pocos programas que actualiza diariamente la información, lo cual aumenta la sensibilidad para la detección de interacciones (15). 
Cuadro 5. Análisis bivariado de la presencia de interacciones

\begin{tabular}{|c|c|c|}
\hline \multirow[t]{2}{*}{ Variable } & \multicolumn{2}{|c|}{ Odds ratio $\left(\mathrm{IC}_{95 \%}\right)$} \\
\hline & $\begin{array}{l}\text { Todas las interacciones } \\
\qquad(n=306)\end{array}$ & $\begin{array}{l}\text { Sin interacciones favorables } \\
\qquad(n=194)\end{array}$ \\
\hline Sexo (femenino Vs. masculino) & $0,95(0,5-1,8), p=0,890$ & $0,99(0,5-1,9), p=0,99$ \\
\hline Edad (años) (>60 Vs. $\leq 60)$ & $3,15(1,6-6,3), p=0,001$ & $2,79(1,3-5,8), p=0,006$ \\
\hline Número de prescriptores (>4 Vs. $\leq 4)$ & $6,42(2,9-14,8), p=0,000$ & $4,65(2,0-10,6), p=0,000$ \\
\hline \multicolumn{3}{|l|}{ Estancia (días) (Vs. 1-3) } \\
\hline $4-6$ & $3,3(1,6-6,7), p=0,001$ & $3,11(1,5-6,6), p=0,003$ \\
\hline$\geq 7$ & $37,3(4,5-278,5), p=0,000$ & $27(3,5-205,5), p=0,001$ \\
\hline \multicolumn{3}{|l|}{ Número de medicamentos ( $V s .=10)$} \\
\hline $\begin{array}{l}11-20 \\
>20\end{array}$ & $\underset{*}{11,7} \underset{*}{(5,5-24,8), p}=0,000$ & $8,4 \underset{*}{(3,8-18,4), p=0,000}$ \\
\hline
\end{tabular}

* Todos los pacientes que recibieron más de 20 medicamentos presentaron por lo menos una interacción, según el análisis bivariado.

Cuadro 6. Análisis de regresión logística múltiple (se muestran solo las variables que retuvieron la significación estadística)

\begin{tabular}{|c|c|c|}
\hline \multirow[t]{2}{*}{ Variable } & \multicolumn{2}{|c|}{ OR $\left(\mathrm{IC}_{95 \%}\right)$} \\
\hline & Todas las interecciones $(n=206)$ & Sin interacciones favorables $(n=146)$ \\
\hline Edad (>60 Vs. $<=60$ años) & $3,1(1,37-7,0), p=0,007$ & $2,9(1,22-6,8), p=0,015$ \\
\hline \multicolumn{3}{|l|}{ Número de medicamentos $(V s .<=10)$} \\
\hline $11-20$ & $11,8(5,4-25,6), p=0,000$ & $8,8(3,9-19,7), p=0,000$ \\
\hline$>20$ & * & * \\
\hline
\end{tabular}

* Todos los pacientes que recibieron más de 20 medicamentos presentaron por lo menos una interacción según la regresión logística múltiple.

En cuanto al tiempo para la aparición de las interacciones, en este estudio la mayoría se presentó tempranamente (en las primeras 24 horas de ingreso a la unidad de cuidados intensivos), lo cual puede obedecer a los cambios terapéuticos que suelen ser necesarios en este periodo debido a las condiciones de ingreso de los pacientes y la gravedad de su condición, que exige una pronta estabilización.

Con respecto al tipo de interacción, las más frecuentes fueron las farmacodinámicas, lo cual difiere de lo reportado por Almeida, et al. (13), en cuyo estudio las interacciones farmacocinéticas fueron las más comunes ( $30 \%$ del total). Este dato es importante, pues evidencia la necesidad de evaluar el uso de un determinado fármaco teniendo en cuenta su mecanismo de acción y los procesos biológicos que modifica, y no solamente las vías metabólicas y de excreción que utiliza.

En cuanto a la gravedad, las interacciones moderadas fueron las más frecuentes, seguidas por las mayores, lo cual concuerda con lo reportado en estudios previos, como el de Rhanna, et al. (17), en el cual se encontró $51 \%$ de interacciones moderadas, o como el de Hammes, et al. (18), que evidenció $68 \%$, y el de Cruciol-Souza (19), en el que se encontró $78 \%$ de interacciones moderadas. Sin embargo, por la diversidad de los programas utilizados para validar las interacciones en los diferentes estudios, es difícil comparar dichos resultados.

Con respecto a interacciones específicas, la más frecuente fue la de fentanilo y midazolam, clasificada como una interacción farmacodinámica con efecto sinérgico; se emplean un analgésico opioide y una benzodiacepina para la analgesia, la sedación y el alivio de la ansiedad en los pacientes con asistencia respiratoria mecánica, de manera que se acomoden al respirador y se optimice la oxigenación, es decir, se usa con un objetivo terapéutico (20). Sin embargo, esta interacción también tiene un componente cinético, ya que el fentanilo inhibe la enzima CYP 3A4, lo cual favorece la acumulación de glucurónido de hidroximidazolam, un metabolito activo del midazolam que se ha encontrado elevado en pacientes con sedación prolongada (21). Esto indicaría que, para lograr una sedación óptima en pacientes críticamente enfermos, es necesario tener presente el potencial de interacciones, emplear escalas validadas para ajustar las dosis, interrumpir diariamente la sedación y diseñar protocolos que eviten el uso prolongado e innecesario de esta combinación (22).

El fentanilo fue uno de los primeros diez fármacos precipitantes e índice, siendo el más frecuente del primer grupo. Ello se explica porque es tanto 
sustrato como inhibidor de la enzima CYP3A4; además, fue el cuarto más prescrito durante el periodo de estudio.

El segundo fármaco precipitante más frecuente fue la dexametasona. Si bien no se ubicó entre los diez más formulados, la dexametasona se comporta como un inductor de la enzima CYP3A4, de manera que puede acelerar el metabolismo de múltiples fármacos, incluidos antihipertensivos como el verapamilo y el diltiazem, inmunosupresores como el tacrolimus, benzodiacepinas, ciertos antifúngicos, y antibióticos como la claritromicina, entre otros (23). El hallazgo del midazolam como el fármaco índice más frecuente puede estar relacionado con su metabolismo, el cual se lleva a cabo mediante la CYP3A4, enzima que es inducida o inhibida por una amplia variedad de fármacos.

Con respecto a los factores asociados con la aparición de las interacciones, el análisis multivariado solo mostró asociación estadísticamente significativa con la edad y el número de medicamentos prescritos. En los análisis adicionales en los que se evaluó la asociación entre la interacción y la duración de la estancia y entre esta y el número de prescriptores según la edad y el número de medicamentos, se evidenció que en las dos asociaciones mencionadas el número de medicamentos se comportó como factor de confusión.

Es interesante que la edad se comporte como un factor de riesgo independiente del número de medicamentos prescrito. Aunque se han documentado cambios farmacocinéticos que desencadenan consecuencias clínicas derivadas de una interacción en ancianos (24), en el caso de las interacciones potenciales, el riesgo en estos pacientes podría deberse al tipo de medicamentos que reciben, pues probablemente con frecuencia son fármacos con mayor probabilidad de precipitar interacciones o de ser modificados por otros.

Que el número de medicamentos apareciera como un factor independiente asociado con la generación de las interacciones, concuerda con lo reportado en la literatura científica en el sentido de que la probabilidad de presentar una interacción medicamentosa depende principalmente del número de fármacos que se prescriban; dicha probabilidad varía entre el 5,6\% para dos medicamentos y más del $80 \%$ en pacientes que reciben siete o más medicamentos $(25,26)$. Cabe destacar que, en la presente investigación, todos los pacientes que recibieron más de 20 medicamentos presentaron por lo menos una interacción.
Los estudios en que se han evaluado posibles factores asociados con la aparición de interacciones en pacientes críticamente enfermos son escasos. Cabe destacar el estudio de Lima, et al. (27), quienes los evaluaron en una cohorte retrospectiva de 102 pacientes de una unidad de cuidados intensivos de adultos. Los autores encontraron que las mujeres tenían una menor probabilidad de interacciones $\left(\mathrm{OR}=0,27 ; \quad \mathrm{IC}_{95 \%} \quad 0,095-0,74\right)$, pero no hallaron una asociación estadísticamente significativa con la edad al comparar a los pacientes de más de 60 años con los de menos edad $\left(\mathrm{OR}=0,94 ; \mathrm{IC}_{95 \%} 0,36-2,47\right)$, ni con la estancia hospitalaria al comparar una de más de nueve días con una de menos días (OR=0,66; $\left.\mathrm{IC}_{95 \%} 0,25-1,7\right)$.

Las diferencias entre estos hallazgos y los obtenidos en el presente estudio pueden explicarse por el tipo de interacciones incluidas (prevalentes en el estudio de Lima, et al., incidentes en este) y por el menor número de pacientes evaluados en el estudio de los primeros. En otro estudio, Mohamed, et al., encontraron que la edad y el número de medicamentos se asociaron con interacciones (28); sin embargo, es difícil establecer comparaciones con el presente estudio porque en aquel se incluyó población pediátrica además de los casos prevalentes.

Al comparar a los pacientes con interacciones mayores y aquellos con interacciones moderadas, se evidenció que los primeros recibían un mayor número de medicamentos, formulados por un mayor número de prescriptores y habían tenido estancias más prolongadas. Sin embargo, estos hallazgos deben interpretarse con cautela, pues no se hizo un análisis formal de factores asociados con interacciones mayores, el cual es importante para detectar a los pacientes en riesgo, por cuanto las interacciones mayores son de relevancia clínica y favorecen la aparición de reacciones adversas a medicamentos.

Las principales fortalezas de este estudio radican en el empleo de varias medidas de incidencia, con lo cual se brindó un panorama más completo de la frecuencia de interacciones durante el periodo de estudio, en comparación con la prevalencia puntual, que es la medida empleada en múltiples estudios sobre las interacciones, así como el empleo de una herramienta validada y sensible para su detección y la inclusión de interacciones incidentes, las cuales generan menos sesgos que los casos prevalentes a la hora de evaluar las asociaciones.

En cuanto a las limitaciones del estudio, cabe mencionar que no se evaluó la asociación entre 
las interacciones y las enfermedades, ya que en la base de datos de la institución frecuentemente se registraban solo los códigos $\mathrm{CIE}-10$ genéricos, lo que dificulta evaluar la asociación con cuadros específicos; tampoco se evaluó la asociación con la gravedad clínica porque no se registraba de manera sistemática una medición objetiva de esta variable, como la que se obtendría utilizando la escala Acute Physiology and Chronic Health Evaluation (APACHE); tampoco fue posible determinar los datos puntuales sobre los prescriptores, por ejemplo, su nivel de entrenamiento, experiencia, y observancia de las guías de práctica clínica, aunque era cierto que la configuración del programa usado para registrar la historia clínica en esta institución implicaba que todas las fórmulas fueran validadas por el especialista de turno.

La ausencia de estas variables pudo generar confusión residual, en especial en la asociación con la edad. Por otra parte, no se evaluó el impacto clínico de las interacciones detectadas, ya que los objetivos se plantearon en términos de interacción potencial; además, el diseño retrospectivo y las fuentes de datos disponibles no permitían obtener una medición válida de este aspecto. Por último, no se evaluaron las interacciones entre fármacos y compuestos nutricionales.

En conclusión, las interacciones farmacológicas son frecuentes en la mayoría de pacientes hospitalizados en las unidades de cuidados intensivos. Si bien algunas interacciones contribuyen a la consecución de los objetivos terapéuticos, otras pueden causar resultados negativos asociados con la medicación. Cuanto mayor sea el número de medicamentos prescritos, mayor es la posibilidad de que se presenten una o varias interacciones. La edad es otro factor que puede estar asociado o, por lo menos, parece ser un marcador de riesgo.

El gran potencial de interacciones en los pacientes críticamente enfermos refuerza la recomendación de evaluar constantemente la necesidad de cada uno de los fármacos prescritos, lo cual implica un ejercicio diario de prescripción razonada y la implementación de ajustes en el tratamiento coherentes con los cambios clínicos del paciente, con lo cual se evita el uso de fármacos innecesarios, la duplicidad y los tratamientos de duración excesiva, y se limita el tiempo de exposición a interacciones potencialmente peligrosas.

Teniendo en cuenta la elevada frecuencia de interacciones farmacológicas durante el cuidado intensivo, y su impacto potencial, sería recomendable que las instituciones faciliten a los médicos tratantes las herramientas sistematizadas y validadas para su detección, con lo cual podrían consultar de forma ágil en caso de dudas sobre posibles interacciones riesgosas entre los medicamentos que prescriben.

Sin embargo, lo ideal sería implementar un sistema de alertas en tiempo real que informe a los médicos sobre posibles interacciones en el momento mismo de hacer la prescripción, en especial sobre las mayores o contraindicadas, de manera que puedan tomar decisiones terapéuticas más seguras e informadas. Una ventaja de tales sistemas es que la alerta es automática y su uso no depende de la voluntad del prescriptor.

La participación de un farmacólogo clínico en el proceso de prescripción de medicamentos es otra medida que puede implementarse para garantizar la detección oportuna de interacciones riesgosas, así como de otros problemas relacionados con la medicación que alteren el curso clínico del paciente, y para que orienten el uso seguro de la medicación, sobre todo en situaciones complejas en las que la medida terapéutica sea imprescindible para el paciente a pesar del riesgo potencial, el cual puede ser controlado.

La instauración efectiva de estas medidas se traduciría en una mejor calidad de la atención y en mayor seguridad para los pacientes.

\section{Conflicto de intereses}

Los autores declaramos que no teníamos conflictos de intereses de orden académico, institucional $u$ operacional en el momento de hacer el estudio.

\section{Financiación}

El primer autor obtuvo el apoyo y la tutoría de los coautores como parte de los requisitos para optar al título de Especialista en Farmacología Clínica de la Universidad de La Sabana. El estudio no contó con financiación de fuente específica.

\section{Referencias}

1. Rodrigues DA, editor. Drug-drug interactions. Second edition. London: Informa Health Care. 2008. p. 768.

2. Pirmohamed M, James S, Meakin S, Green C, Scout A, Walley $\mathrm{T}$, et al. Adverse drug reactions as cause of admission to hospital: Prospective analysis of 18.820 patients. BMJ. 2004; 329:15-9. https://doi.org/10.1136/bmj. 329.7456.15

3. Pouyanne $\mathbf{P}$, Haramburu F, Imbs J, Bégaud B. Admissions to hospital caused by adverse drug reactions: Cross sectional incidence study. BMJ. 2000;320:1036-45. https:// doi.org/10.1136/bmj.320.7241.1036 
4. Becker ML, Kallewaard M, Caspers PW, Visser LE, Leufkens HG, Stricker BH. Hospitalizations and emergency department visits due to drug-drug interactions: A literature review. Pharmacoepidemiol Drug Saf. 2007;16: 641-51. https://doi.org/10.1002/pds.1351

5. Caribé RA, Chaves GR, Pocognoni JD, Souza IA. Potenciales interacciones medicamentosas en pacientes con sepsis internados en la unidad de terapia intensiva. Farm Hosp. 2013;37:383-7. https://doi.org/10.7399/FH. 2013.37.5.534

6. Rivkin A, Yin H. Evaluation of the role of the critical care pharmacist in identifying and avoiding or minimizing significant drug-drug interactions in medical intensive care patients. J Crit Care. 2011;26:104.e1-6. https://doi. org/10.1016/j.jcrc.2010.04.014

7. Reis AM, Cassiani SH. Prevalence of potential drug interactions in patients in an intensive care unit of a university hospital in Brazil. Clinics (Sao Paulo). 2011;66:915. https://doi.org/10.1590/S1807-59322011000100003

8. Plaza J, Álamo M, Torres P, Fuentes A, López F. Interacciones de medicamentos y eventos adversos en fármacos utilizados en una unidad de cuidados intensivos. Rev Med Chile. 2010;138 452-60. https://doi.org/10.4067/ S0034-98872010000400009

9. Bergk V, Gasse C, Rothenbacher D, Loew M, Brenner H, Haefeli WE. Drug interactions in primary care: Impact of a new algorithm on risk determination. Clin Pharmacol Ther. 2004;76:85-96. https://doi.org/10.1016/j.clpt.2004.02.009

10. Askari M, Eslami S, Louws M, Wierenga PC, Dongelmans DA, Kuiper RA, et al. Frequency and nature of drug-drug interactions in the intensive care unit. Pharmacoepidemiol Drug Saf. 2013;22:430-7. https://doi.org/10.1002/pds.3415

11. Smithburger P, Kane-Gill SL, Seybert AL. Drugdrug interactions in the medical intensive care unit: An assessment of frequency, severity and the medications involved. Int J Pharma Pract. 2012;20:402-8. https://doi. org/10.1111/j.2042-7174.2012.00221.x

12. Institute of Medicine (US) Committee on Quality of Health Care in America; Kohn LT, Corrigan JM, Donaldson MS, editors. To err is human: Building a safer health system. Washington (DC): National Academies Press (US); 2000. Fecha de consulta: 17 de febrero de 2017. Disponible en: https://www.ncbi.nlm.nih.gov/books/NBK225182/

13. Almeida SM, Gama SC, Akamine N. Prevalence and classification of drug-drug interactions in intensive care patients, Einstein (Sao Paulo). 2007;5:347-51.

14. McFarland HM. Identification and management of drug interactions. Medscape. Fecha de consulta: 20 de mayo de 2016. Disponible en: http://www.medscape.org/viewarticle/ 418376

15. Joseph A. Khan A. A comparison of the Micromedex vs. Lexicomp medicine information databases. Fecha de consulta: 20 de mayo de 2016. Disponible en: http://www. ukmi.nhs.uk/filestore/misc/AcomparisonoftheMicromedexa ndLexi-compDatabases.pdf

16. Barrons R. Evaluation of personal digital assistant software for drug interactions. Am J Health Syst Pharm. 2004;61:380-5.

17. Carvalho RE, Reis AM, Faria LM, ZagoKS, Cassiani SH. Prevalence of drug interactions in intensive care units in Brazil. Acta Paul Enferm. 2013;26:150-7.

18. Hammes J, Pfuetzenreiter F, daSilveira F, Koenig A, AdrienoWestphal G. Potential drug interactions prevalence in intensive care units. Rev Bras Ter Intensiva. 2008;20:34954. https://doi.org/10.1590/S0103-507X2008000400006

19. Cruciol-Souza JM, Thomson JC. Prevalence of potential drug-drug interactions and its associated factors in a Brazilian teaching hospital. J Pharm Pharm Sci. 2006;9:427-33.

20. Devlin JW, Roberts RJ. Pharmacology of commonly used analgesics and sedatives in the ICU: Benzodiazepines, propofol, and opioids. Crit Care Clin. 2009;25:431-49. https://doi.org/10.1016/j.ccc.2009.03.003

21. Riker RR, Fraser GL. Altering intensive care sedation paradigms to improve patient outcomes. Crit Care Clin. 2009;25:527-38. https://doi.org/10.1016/j.ccc.2009.05.004

22. Schweickert WD, Kress JP. Strategies to optimize analgesia and sedation. Crit Care. 2008;12(Suppl.3):S6. https:// doi.org/10.1186/cc6151

23. López JM. Metabolismo de fármacos: enzimas CYP3A (sub familia del citocromo P450). Fecha de consulta: 21 de marzo de 2013. Disponible en: http://www.info-farmacia. com/bioquimica/metabolismo-de-farmacos-enzimas-cyp3asub-familia-del-citocromo-p450

24. Hansten PD. Drug interaction management. Pharm World Sci. 2003;25:94-7. https://doi.org/10.1023/A:1024077018902

25. Elanjian S, Gora ML, Symes LR. Methods used by pharmacy departments to identify drugs interactions. Am J Hosp Pharm. 1993;50:2546-9.

26. Bucşa C, Farcaş A, Cazacu I, Leucuta D, AchimasCadariu A, Mogosan C, et al. How many potential drug-drug interactions cause adverse drug reactions in hospitalized patients? Eur J Intern Med. 2013;24:27-33. https://doi.org/10.1016/j.ejim.2012.09.011

27. Lima RE, Cassiani SH. Potential drug interactions in intensive care patients at a teaching hospital. Rev Lat Am Enfermagem. 2009;17:222-7. https://doi.org/10.1590/ S0104-11692009000200013

28. El Samia Mohamed SMA, Gad ZM, El-Nimr NA, Abdel Razek AAH. Prevalence and pattern of potential drug-drug interactions in the critical care units of a tertiary hospital in Alexandria, Egypt. Adv Pharmacoepidemiol Drug Saf. 2013;2:144-54. https://doi.org/10.4172/2167-1052.1000144 